\title{
Search for top squark pair production and decay in four bodies, with two leptons in the final state, at the ATLAS Experiment with LHC Run2 data
}

\author{
Marilea Reale* \\ INFN Lecce and Università del Salento (IT) \\ E-mail: marilea.reale@cern.ch \\ Luigi Longo* \\ INFN Lecce and Università del Salento (IT) \\ E-mail: luigi.longo@cern.ch
}

\begin{abstract}
Supersymmetry (SUSY) still remains one of the most interesting theories which are candidates to describe physics beyond the Standard Model, even if the latest Run1 results and interpretations have so far shown no experimental evidence for the existence of superparticles. If SUSY exists, the strong production of colored SUSY particles is expected to be the dominant production process at the LHC. In this work a search for the top quark supersymmetric partner (stop) pairs production is reported, using $36.1 \mathrm{fb}^{-1}$ of proton-proton collision data collected by the ATLAS experiment in 2015 and 2016. The analysis targets the stop decaying, with $100 \%$ branching ratio, into a b quark, a neutralino (here assumed to be the lighest supersymmetric particle), a fermion and an antifermion, with a final state including two isolated electrons or muons and missing transverse momentum. The results are interpreted in the stop and neutralino mass plane in terms of exclusion limits, since no excess in data has been found for this analysis.
\end{abstract}

The European Physical Society Conference on High Energy Physics

5-12 July

Venice, Italy

${ }^{*}$ on behalf of the ATLAS Collaboration 


\section{Introduction}

Supersymmetry (SUSY) is one of the most popular and promising extensions for the Standard Model theory, aiming to solve many open questions known in particle physics. Even if no experimental evidence for the existence of SUSY particles (superparticles) have been obtained from the LHC Run1 results, the strong production of colored superparticles is expected to be the dominant process at the LHC.

In most R-parity ${ }^{1}$ conserving SUSY models the partners of the third generation quarks are typically lighter than the partners of the first and second generation quarks. In addition, considerations of naturalness suggest that the light supersymmetric partners of the top quark (the so-called top squark or stop, $\tilde{t}_{1}$ ) cannot be too heavy, to keep the Higgs boson mass close to the electroweak scale and could be thus produced with a relatively large cross section at the LHC.

Stop can decay into a variety of final states, depending, amongst other factors, on the hierarchy and mixing of the SUSY partners of the Higgs and of the electroweak gauge bosons.

For direct stop-pair production, with each stop decaying into a top quark and the lightest neutralino $^{2} \tilde{\chi_{1}^{0}}$ (assumed to be the lightest supersymmetric particle, LSP), a particularly interesting and still unexcluded region is the one defined by the boundaries $m_{\tilde{t}}=m_{\tilde{\chi}_{1}^{0}}$ from the left and $m_{\tilde{t}}-m_{\tilde{\chi}_{1}^{0}}=m_{b}+m_{W}$ from the right. One of the possible decay channels for the stop, in this region, is the four-body decay, $\tilde{t}_{1} \rightarrow b f f^{\prime} \tilde{\chi}_{1}^{0}$, where $f$ and $f^{\prime}$ are the fermion and the antifermion coming from the $W^{*}$ decay.

In this paper, an analysis seeking stop-pair production in final states with large missing transverse energy $\left(E_{T}^{\text {Miss }}\right.$ ) and two soft leptons (electron $e$ or muon $\mu$ ) is presented [1] and, for this study, a simplified model is considered, in which the stop is assumed to decay into four-body with $100 \%$ branching ratio. The shown results are obtained using $36.1 \mathrm{fb}^{-1}$ of $\sqrt{s}=13 \mathrm{TeV}$ proton-proton collisions recorded by the ATLAS [2] experiment at the LHC in 2015 and 2016.

\section{Analysis strategy}

Event selection has been implemented aiming to reproduce the targeted final state signature and optimized in order to maximize the analysis sensitivity.

The kinematics of the four-body decay of the $\tilde{t}$ varies rapidly as one moves between the two kinematic boundaries. Where $m_{\tilde{t}}$ is adequately greater than $m_{\tilde{\chi}_{1}^{0}}$, there is enough phase-space for the two leptons to be produced (although both being soft) and efficiently resolved in the final state. Otherwise, moving toward the $m_{\tilde{t}}=m_{\tilde{\chi}_{1}^{0}}$ line, the phase-space becomes more limited and the selection on the two soft leptons brings this analysis to lower sensitivity.

To allow for a selection of events with soft leptons, triggers based on $E_{T}^{\text {Miss }}$ have been used.

Events containing exactly two opposite-sign signal leptons of either the same flavor $(e e, \mu \mu)$ or different flavor $(e \mu)$ are selected. Since the two leptons are expected to be soft, upper limits on their $p_{T}$ of $80 \mathrm{GeV}$ and $35 \mathrm{GeV}$ are applied for the leading and the sub-leading leptons, respectively.

As the phase-space is anyway limited, the LSP's are typically soft, meaning that their contribution

\footnotetext{
${ }^{1} R=(-1)^{3(B-L)+2 S}$, where $B$ is the baryon number, $L$ is the leptonic number and $S$ is the spin of the particle.

${ }^{2}$ Neutralino is defined as the mass eigenstates obtained by mixing the neutral supersymmetric partner of the Higgs and the electroweak gauge bosons.
} 
to the $E_{T}^{\text {Miss }}$ in the event can be small. The final state, therefore, is the same as for the production and decay of pairs of top quarks $(t \bar{t})$, which constitutes the dominant background to the signal process under the study.

However, the request of an energetic initial state radiation (ISR) jet seems to help in discriminating signal against background events, since the boosted events show an enhanced value of $E_{T}^{\text {Miss }}$.

Thus, at least 2 jets are requested in the final state, and the leading jet of each event is considered as an ISR jet candidate and is required to have $p_{T}>150 \mathrm{GeV}$. However, as in this particular phasespace region the jets resulting from stop decays tend to be soft, at most one more energetic jet with $p_{T}>25 \mathrm{GeV}$ is permitted in the event and the transverse momentum of the third jet (if present) must satisfy $p_{T}^{\text {jet }} / E_{T}^{\text {Miss }}<0.14$.

Only the events in which the invariant mass of the two leptons is greater than $10 \mathrm{GeV}$ are accepted in order to remove leptons from low mass resonances. Further, a requirement of $E_{T}^{M i s s}>200 \mathrm{GeV}$ is imposed, in order to be on the trigger efficiency plateau.

The signal region (SR) definition is then optimized requiring $R_{2 l}>12$ and $R_{2 l 4 j}>0.35$, where $R_{2 l}=E_{T}^{M i s s} /\left(p_{T}^{l e p_{1}}+p_{T}^{l e p_{2}}\right), R_{2 l 4 j}=E_{T}^{\text {Miss }} /\left(m_{\text {eff }}\right)$ and $m_{\text {eff }}$ is defined as the scalar sum of the $E_{T}^{\text {Miss }}$ and the transverse momenta of the two leptons and of the hadronic activity up to 4 jets. The last two cuts are of particular importance in discriminating the signal against the $t \bar{t}$ background.

Finally, in the entire region, due to the low values of $\Delta m$, the two b-jets produced in the $\tilde{t}$ decays are rarely tagged as coming from a $b$ quarks. On the contrary, a veto on b-tagged jets is applied in order to strongly reduce the contribution from $t \bar{t}$ events.

The contribution of the dominant irreducible SM backgrounds, i.e. the $t \bar{t}$ production, the diboson production and the $\mathrm{Z}$ boson decaying into $\tau \tau$ processes, is evaluated by constraining the normalisation of their yields with observed data in dedicated respective background dominated control regions (CRs), and then extrapolating these yields to the SR. Normalization factors (SFs) obtained from the fits of CRs are validated in dedicated, so called, validation regions (VRs).

The background arising from fake leptons (non-prompt leptons produced in semileptonic hadron decays and from jets showing a lepton signature) has non-negligible rates in the SRs and CRs considered in this analysis due to the very low transverse momentum threshold in the leptons selection. The fake rates are estimated by means of a data-driven technique similar to that described in Refs. $[3,4]$.

Minor backgrounds yields are taken directly from Monte Carlo (MC) simulation.

\section{Results and interpretation}

Figure 1 shows the data/MC comparison for two of the most meaningful variables when all the SR cuts are applied except for the cut on the shown variables and when the SFs are applied on MC prediction. No significant excess can be observed: data are compatible with SM prediction within 1 standard deviation and this is reflected in the background-only fit results for the SR, shown in Table 1. 

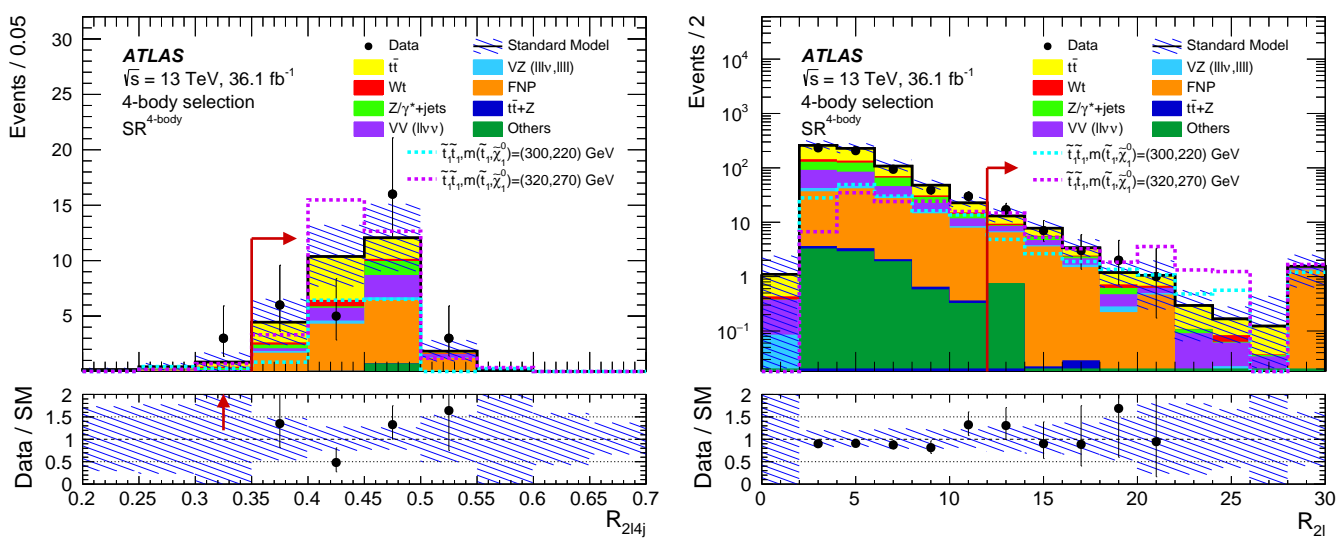

Figure 1: Four-body selection distributions of (a) $R_{2 l 4 j}$ and (b) $R_{2 l}$ for events satisfying all the SR selections except for the one on the variable shown in the figure, after the background fit. The contributions from all SM backgrounds are shown as a histogram stack; the hatched bands represent the total statistical and systematic uncertainty. The rightmost bin of each plot includes overflow events. Reference top squark pair production signal models are overlayed for comparison. Red arrows indicate the signal region selection criteria [1].

Table 1: Four-body selection background fit results for SR. The nominal predictions from MC simulation are given for comparison for those backgrounds $\left(t \bar{t}, \mathrm{VV}\right.$ and $\left.Z_{\tau \tau}\right)$ that are normalised to data in dedicated CRs. The "Others" category contains the contributions from $t \bar{t} W, t \bar{t} h, t \bar{t} W W, t \bar{t} t, t \bar{t} t \bar{t}, W h, g g h$ and $Z h$ production. Combined statistical and systematic uncertainties are given [1].

\begin{tabular}{lr}
\hline & SR \\
\hline Observed events & 30 \\
\hline Estimated SM Events & $28 \pm 6$ \\
\hline$t \bar{t}$ & $7.9 \pm 2.0$ \\
$V V$ & $4.5 \pm 2.3$ \\
$Z_{\tau \tau}$ & $1.2 \pm 0.6$ \\
$t \bar{t}+Z$ & $0.03 \pm 0.01$ \\
$W t$ & $1.08 \pm 0.27$ \\
$Z_{e e}, Z_{\mu \mu}$ & $0.21 \pm 0.09$ \\
Others & $0.80 \pm 0.30$ \\
Fake and non-prompt & $12.8 \pm 4.3$ \\
\hline Fit input, $t \bar{t}$ & 7.7 \\
Fit input, $V V$ & 5.7 \\
Fit input, $Z_{\tau \tau}$ & 1.1 \\
\hline
\end{tabular}

Model-independent upper-limits have been estimated on the visible cross-section $\left(\sigma_{\mathrm{vis}}\right)$ : the computed upper limit for the $\sigma_{\mathrm{vis}}$ is $0.48 \mathrm{fb}$, corresponding to discovery p-value $p(s=0)=0.37$. Model-dependent exclusion limits have also been set in the stop and neutralino mass parameters plane as shown in Figure 2. 


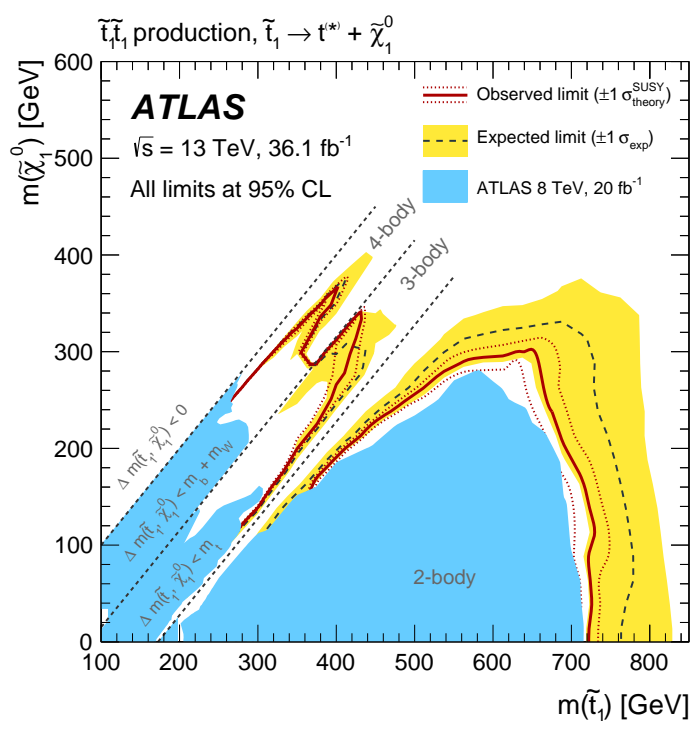

Figure 2: Exclusion contour for a simplified model assuming $\tilde{t}_{1}$ pair production, decaying via $\tilde{t}_{1} \rightarrow t^{(*)} \tilde{\chi}_{1}^{0}$ with $100 \%$ branching ratio. The dashed grey line and the shaded yellow band are the expected limit and its $\pm 1 \sigma$ uncertainty. The thick solid red line is the observed limit for the central value of the signal crosssection. The expected and observed limits do not include the effect of the theoretical uncertainties in the signal cross-section. The dotted lines show the effect on the observed limit when varying the signal crosssection by $\pm 1 \sigma$ of the theoretical uncertainty [1].

\section{References}

[1] ATLAS Collaboration, Search for direct top squark pair production in final states with two leptons in $\sqrt{s}=13 \mathrm{TeV}$ pp collisions with the ATLAS detector, arXiv:1708.03247 [hep-ex].

[2] ATLAS Collaboration, 2008 JINST 3 S08003.

[3] ATLAS Collaboration, Measurement of the top quark-pair production cross section with ATLAS in pp collisions at $\sqrt{s}=7 \mathrm{TeV}$, Eur. Phys. J. C 71 (2011) 1577, DOI:10.1140/epjc/s10052-011-1577-6.

[4] ATLAS Collaboration, Measurement of the top quark pair production cross section in pp collisions at $\sqrt{s}=7 \mathrm{TeV}$ in dilepton final states with ATLAS, Phys. Lett. B 707 (2012) 459,

DOI:10.1016/j.physletb.2011.12.055. 